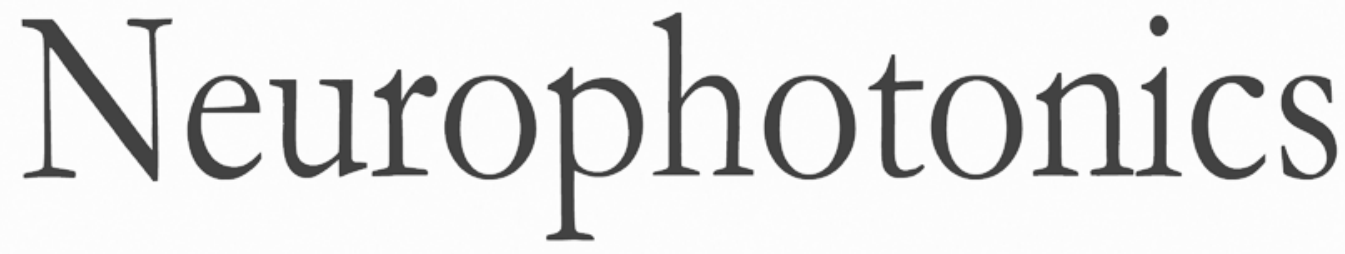

\title{
Noninvasive optical assessment of resting-state cerebral blood flow in children with sickle cell disease
}

Seung Yup Lee

Kyle R. Cowdrick

Bharat Sanders

Eashani Sathialingam

Courtney E. McCracken

Wilbur A. Lam

Clinton H. Joiner

Erin M. Buckley 


\title{
Noninvasive optical assessment of resting-state cerebral blood flow in children with sickle cell disease
}

\author{
Seung Yup Lee, ${ }^{\text {a }}$ Kyle R. Cowdrick, ${ }^{\text {a }}$ Bharat Sanders, ${ }^{a}$ Eashani Sathialingam, ${ }^{\text {a }}$ Courtney E. McCracken, ${ }^{b}$ \\ Wilbur A. Lam, ${ }^{a, b}$ Clinton H. Joiner, ${ }^{b, c}$ and Erin M. Buckley ${ }^{a, b, d, *}$ \\ ${ }^{a}$ Georgia Institute of Technology and Emory University, Wallace H. Coulter Department of Biomedical Engineering, Atlanta, Georgia, United States \\ ${ }^{\mathrm{b}}$ Emory University School of Medicine, Department of Pediatrics, Atlanta, Georgia, United States \\ 'Children's Healthcare of Atlanta, Aflac Cancer and Blood Disorders Center, Atlanta, Georgia, United States \\ 'Children's Healthcare of Atlanta, Children's Research Scholar, Atlanta, Georgia, United States
}

\begin{abstract}
Sickle cell disease (SCD) is a genetic blood disorder that has profound effects on the brain. Chronic anemia combined with both macro- and microvascular perfusion abnormalities that arise from stenosis or occlusion of blood vessels increased blood viscosity, adherence of red blood cells to the vascular endothelium, and impaired autoregulatory mechanisms in SCD patients all culminate in susceptibility to cerebral infarction. Indeed, the risk of stroke is 250 times higher in children with SCD than in the general population. Unfortunately, while transcranial Doppler ultrasound (TCD) has been widely clinically adopted to longitudinally monitor macrovascular perfusion in these patients, routine clinical screening of microvascular perfusion abnormalities is challenging with current modalities (e.g., positron emission tomography and magnetic resonance imaging) given their high-cost, requirement for sedation in children $<6$ year, and need for trained personnel. We assess the feasibility of a low-cost, noninvasive optical technique known as diffuse correlation spectroscopy (DCS) to quantify an index of resting-state cortical cerebral blood flow (BFI) in 11 children with SCD along with 11 sex- and age-matched healthy controls. As expected, BFI was significantly higher in SCD subjects compared to healthy controls $(p<0.001)$. Within SCD subjects, BFI was inversely proportional to resting-state arterial hemoglobin levels $(p=0.012)$, consistent with expected anemia-induced compensatory vasodilation that aims to maintain adequate oxygen delivery to the tissue. Further, in a subset of patients measured with TCD $(n=7)$, DCSmeasured blood flow was correlated with TCD-measured blood flow velocity in middle cerebral artery $\left(R_{s}=\right.$ $0.68)$, although the trend was not statistically significant $(p=0.11)$. These results are consistent with those of several previous studies using traditional neuroimaging techniques, suggesting that DCS may be a promising low-cost tool for assessment of tissue-level CBF in pediatric SCD. ๑ The Authors. Published by SPIE under a Creative Commons Attribution 4.0 Unported License. Distribution or reproduction of this work in whole or in part requires full attribution of the original publication, including its DOI. [DOI: 10.1117/1.NPh.6.3.035006]
\end{abstract}

Keywords: sickle cell disease; cerebral blood flow; diffuse correlation spectroscopy.

Paper 19025R received Mar. 12, 2019; accepted for publication Jul. 24, 2019; published online Aug. 19, 2019.

\section{Introduction}

Sickle cell disease (SCD) is a genetic blood disorder affecting nearly 300,000 neonates worldwide per year. ${ }^{1}$ A single mutation in the beta-globin gene produces sickle hemoglobin ( $\mathrm{HbS})$, a structural variant of normal adult hemoglobin (HbA). While patients with sickle cell trait who inherit one $\mathrm{HbS}$ gene and one $\mathrm{HbA}$ gene are typically asymptomatic, individuals who inherit $\mathrm{HbS}$ genes from both parents suffer from sickle cell anemia that can cause serious acute and chronic complications in multiple organs. $^{2}$

The cerebral effects of SCD are substantial. By age 15, 2\% of pediatric SCD patients experience an overt stroke accompanied by obvious neurological symptoms, and $\sim 40 \%$ of patients will develop silent infarctions. ${ }^{3}$ These silent infarcts accumulate occultly, but they can be seen by MRI and are associated with cognitive impairments and increased risk of overt stroke. ${ }^{3-6}$ Moreover, compared with age-matched controls, children with SCD have significantly diminished IQ, increased incidence of attention deficits, and impaired executive skills and visuospatial memory. ${ }^{3,7-9}$

*Address all correspondence to Erin M. Buckley, E-mail: erin.buckley@ emory.edu
Although the exact mechanisms of brain injury in these patients are not thoroughly understood, stroke and subsequent neurological impairments in children with SCD are generally believed to stem from the presence of chronic anemia coupled with perfusion abnormalities that arise from stenosis or occlusion of blood vessels, increased blood viscosity, adherence of red blood cells to the vascular endothelium, and impaired autoregulatory mechanisms. ${ }^{10,11}$ Chronic anemia leads to a persistent global hyperemia in order to preserve adequate oxygen delivery to the tissue. ${ }^{12-16}$ Unfortunately, hyperemic vasodilation, impaired cerebrovascular autoregulation, and limited vascular reserve $\mathrm{e}^{17-19}$ leave the brain vulnerable and ill-equipped to respond to increased metabolic demand or reduced blood oxygen content; consequently, the tissue is susceptible to infarction. ${ }^{20}$

Transcranial Doppler ultrasound (TCD) is the standard screening tool for overt stroke risk in pediatric SCD. ${ }^{21-23}$ Through timely initiation of transfusion therapy in patients whose TCD-measured middle cerebral artery blood flow velocity $(\mathrm{CBFV})$ is $>200 \mathrm{~cm} / \mathrm{s}$, the risk of overt stroke in children has significantly diminished by more than $80 \%$.,22 Despite this success, multiple limitations of TCD remain: (1) TCD is insensitive to silent infarctions, (2) some overt strokes are still observed in patients with $\mathrm{CBFV}<200 \mathrm{~cm} / \mathrm{s}^{21,24}$ (3) overt 
strokes do not occur in $\sim 75 \%$ of children with $\mathrm{CBFV}>$ $200 \mathrm{~cm} / \mathrm{s}$, submitting them to unnecessary transfusion therapy, ${ }^{21}$ and (4) TCD has not proven to be useful in assessing safe guidelines for cessation of transfusion therapy, a treatment that is burdensome to patients and families and is also associated with risk of infection, iron overload, and alloimmunization. ${ }^{25,26}$ Supplementary modalities to TCD are needed for improved cerebral monitoring in SCD patients in order to increase specificity in stroke screening and to initiate timely therapeutic interventions.

Measurements of microvascular, tissue-level CBF have shown promise as complementary data to TCD measurements of macrovascular CBFV in SCD. Several investigations have demonstrated that resting-state cortical CBF in SCD is significantly elevated compared to age-matched controls, ${ }^{14-16,27}$ even in the presence of seemingly normal TCD velocities. ${ }^{13,28}$ Indeed, numerous reports have shown a mismatch between macrovascular blood flow velocities and microvascular blood flow, as indicated by a lack of correlation between TCD measured CBFV and cerebral blood flow. ${ }^{13,29}$ Moreover, perfusion abnormalities in SCD have been associated with increased incidence of neurological symptoms (e.g., reversible ischemic neurological deficit and transient ischemic attack) and overt stroke, ${ }^{10,30}$ and elevated CBF has been associated with lower IQ. ${ }^{13}$ Further, recent work has demonstrated that transfusions act to reduce $\mathrm{CBF}$ and relieve metabolic demand. ${ }^{31}$ Taken together, these studies suggest that identification of microvascular CBF abnormalities may improve characterization of stroke risk, guide treatment strategies, and possibly help mitigate adverse neurocognitive outcomes in children with SCD..$^{10,13,14,32}$ Unfortunately, current tissue-level CBF neuroimaging tools (e.g., PET and MRI) are prohibitively expensive, have limited access, require anesthesia in children $<6$ year, and sometimes rely on external contrast agents, making them inappropriate for the routine clinical tools or use in countries with low-resource settings.

Herein, we assess the feasibility of a low-cost, noninvasive optical technique, known as diffuse correlation spectroscopy (DCS), to quantify microvascular cerebral blood flow in pediatric patients with SCD. DCS uses near-infrared light to relate intensity fluctuations of multiply scattered reflected light detected at the tissue surface to a blood flow index (BFI) in the underlying tissue. ${ }^{33,34}$ DCS has been extensively validated as a trend monitor of relative change in CBF over time (as recently reviewed by Refs. 35 and 36). However, resting-state BFI has only be validated clinically in neonates and small animals, as the presence of a thick $(\geq 1 \mathrm{~cm})$ extracerebral layer in older patients can significantly confound estimations of absolute BFI and lead to substantial errors. Nevertheless, our results suggest that in children with SCD, the alterations in cerebral blood flow appear to be substantial enough to significantly reduce the effects of extracerebral layers such that we are able to observe expected CBF trends seen by other "gold standard" modalities, i.e., (1) the DCS measured BFI is increased in children with SCD compared to healthy controls and (2) within SCD subjects, BFI is inversely correlated with arterial hemoglobin concentration.

\section{Materials and Methods}

\subsection{Subject Cohort}

Children ages 2 to 10 years old with $\mathrm{SCD}$ ( $\mathrm{HbSS}$ or $\mathrm{HbS} \beta^{0}$ thalassemia) along with healthy age- and sex-matched controls were enrolled at Children's Healthcare of Atlanta. Subjects were excluded if they had a significant acute illness within 1 month of optical assessment, were hypertensive, had a history of major head injury requiring a visit to an emergency department, seizure disorder, or prenatal or perinatal hypoxic-ischemic brain injury. Written informed consent was obtained from legal guardians of all participants. The study was approved by the Children's Healthcare of Atlanta Institutional Review Board.

Phlebotomy was performed on all SCD subjects for a clinically indicated complete blood count (including hemoglobin, hematocrit, red blood cell count, white blood cell count, and reticulocyte count) within 2 days of optical measurements. A complete clinically indicated TCD assessment was also performed in a subset of SCD subjects $(n=15)$ within 1 day of optical measurements.

\subsection{DCS Measurement of Blood Flow}

DCS utilizes near-infrared light to noninvasively estimate microvascular blood flow. In DCS, the tissue is illuminated with a long-coherence laser, and temporal fluctuations in reflected light intensity are measured by a remotely located detector. These intensity fluctuations are primarily caused by the motion of red blood cells. To relate intensity fluctuations to red blood cell flow in the interrogated tissue volume, we compute an intensity autocorrelation function, $g_{2}(\tau)$. Then, we fit $g_{2}(\tau)$ to a simple analytical model, known as correlation diffusion theory, to extract a BFI $\left(\mathrm{cm}^{2} / \mathrm{s}\right)$. This BFI reflects the average flow of red blood cells within the interrogated tissue volume. ${ }^{33,34,37,38}$

For the experiments described herein, we employed a custom-built DCS system that consisted of an 852-nm longcoherence laser (iBeam Smart, TOPTICA Photonics, Farmington, New York), two four-channel single photon counting modules (SPCMAQ4C-IO, Perkin-Elmer, Quebec, Canada), and an eight-channel hardware correlator (Flex05-8ch, ${ }^{39} \mathrm{New}$ Jersey). For subject measurements, we used a DCS sensor with two source-detector separations [1.0 and $2.5 \mathrm{~cm}$, Fig. 1(b)] for varying depth sensitivities [Fig. 1(c)]. ${ }^{40}$ All fibers were embedded in a rubber-like flexible holder. Given an approximate depth to the brain of $1 \mathrm{~cm}$ in sickle cell patients of this age range, the short separation $(r=1 \mathrm{~cm})$ is primarily sensitive to extracerebral tissue layers, while the longer separation $(r=2.5 \mathrm{~cm})$ is sensitive to both cerebral and extracerebral layers. ${ }^{40}$ The sensor consisted of a 1-mm source fiber bundle (50- $\mu \mathrm{m}$ multimode fibers, NA $=0.66$, FTTIIG23767, Fiberoptics Technology, Pomfret, Connecticut), a $4.4-\mu \mathrm{m}$ singlemode fiber (780HP, Thorlabs) at the $1-\mathrm{cm}$ separation, and seven single-mode fibers bundled together at the $2.5-\mathrm{cm}$ separation to compensate for the exponential decrease in detected light intensity with increasing source-detector separation. The detected autocorrelation curves from these seven detectors at $2.5 \mathrm{~cm}$ were averaged for improved signal-to-noise ratio (SNR). Care was taken to ensure compliance with the American National Standards Institute (ANSI) maximum permissible exposure of skin to laser radiation $\left(<4 \mathrm{~mW} / \mathrm{mm}^{2}\right.$ at $\left.852 \mathrm{~nm}\right)$. Specifically, we manually adjusted the laser power such that the power output of the optical sensor was $\sim 40 \mathrm{~mW}$. Further, we ensured a spot size of radius $>2 \mathrm{~mm}$ by either putting a spacer or a $5-\mathrm{mm}$ rightangle-prism between the source fiber tip and the tissue surface.

For each measurement session, the DCS sensor was secured on either right or left forehead for $\sim 2 \mathrm{~min}$ of $0.3-\mathrm{Hz}$ data acquisition [Fig. 1(a)], resulting in 40 to 50 intensity autocorrelation measurements. In a subset of subjects $(n=23)$, we also employed a frequency-domain near-infrared spectroscopy 
(a)

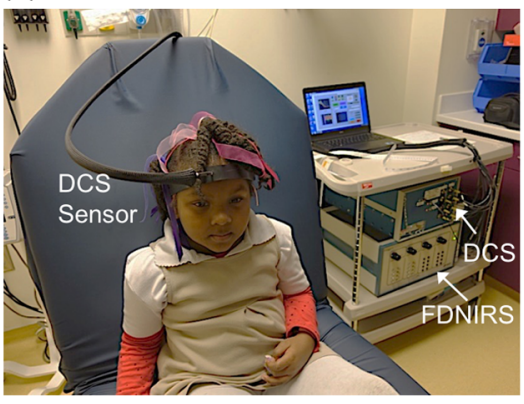

(b)

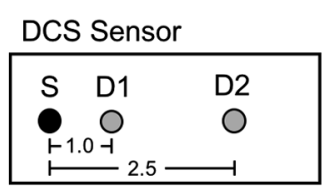

(c)

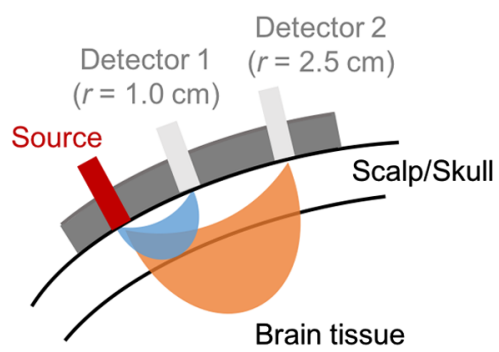

Fig. 1 Measurement setup (a) DCS data were acquired using fiber-optic-based sensor secured on the forehead, (b) source-detector geometry of the DCS probe, (c) schematic of the optical interrogation path formed by the 1 and $2.5 \mathrm{~cm}$ source-detector separations in the DCS sensor. The short separation $(r=1 \mathrm{~cm})$ is primarily sensitive to extracerebral layers, while the longer separation $(r=2.5 \mathrm{~cm})$ is sensitive to both cortical tissues and extracerebral layers.

Table 1 Demographic characteristics of the study population included for DCS-measured cerebral blood flow data analysis.

\begin{tabular}{|c|c|c|c|}
\hline & SCD & Control & $p$ \\
\hline$n$ & 11 & 11 & \\
\hline Age $(y)$ & 6.9 (5.5 to 8.2$)$ & 7.3 (5.7 to 8.1$)$ & 0.87 \\
\hline $\begin{array}{l}\text { Gender } \\
\text { (male:female) }\end{array}$ & $4: 7$ & $4: 7$ & 1.0 \\
\hline Weight (kg) & 21.8 (20.2 to 23.5 ) & 22.1 (21.2 to 28.5 ) & 0.32 \\
\hline Height $(\mathrm{cm})$ & 115.2 (113.3 to 121.2$)$ & $120.3(118.4$ to 125.1$)$ & 0.15 \\
\hline BMI $\left(\mathrm{kg} / \mathrm{cm}^{2}\right)$ & 15.8 (14.5 to 16.1$)$ & 15.3 (14.6 to 17.1$)$ & 1.0 \\
\hline $\begin{array}{l}\text { Head } \\
\text { circumference } \\
(\mathrm{cm})\end{array}$ & 51.0 (49.6 to 52.0$)$ & 52.0 (52.0 to 53.0$)$ & 0.18 \\
\hline
\end{tabular}

Note: Data presented as median (interquartile range, Q1 to Q3), BMI, body mass index

(FDNIRS) to assess resting-state tissue optical properties for improving DCS analysis (see Sec. 6).

\subsection{DCS Data Analysis}

For DCS analysis, the measured intensity autocorrelation functions, $g_{2}(\tau)$ ), were first evaluated for signal quality. Data were discarded according to the following predefined exclusion criteria: (1) intensity $<8 \mathrm{kHz}$, and/or $(2) g_{2}(\tau=1 \mathrm{~s})>1.005$, indicative of motion artifact. Next, $g_{2}(\tau)$ at both 1 and $2.5 \mathrm{~cm}$ were each fit for a BFI using the semi-infinite solution to the correlation diffusion equation. ${ }^{37}$ Fits were constrained to $g_{2}(r=1 \mathrm{~cm}, \tau)>1.005$ and $g_{2}(r=2.5 \mathrm{~cm}, \tau)>1.2$ to increase sensitivity to the extracerebral and cerebral layers, respectively. ${ }^{40,41}$ For all these fits, we assumed an index of refraction of 1.4, and we input the FDNIRS-estimated reduced scattering $\left(\mu_{\mathrm{s}}^{\prime}\right)$ and absorption $\left(\mu_{\mathrm{a}}\right)$ coefficients at $852 \mathrm{~nm}$ of the corresponding hemisphere in which DCS was measured (see Sec. 6). If FDNIRS measurements were not available, group averaged scattering and absorption coefficients were used (see Sec. 6). Finally, the measured BFI across the 2 min monitoring period $(0.3 \mathrm{~Hz})$ were averaged to yield a mean BFI at $r=1$ and
$2.5 \mathrm{~cm}$, dubbed $\mathrm{BFI}_{1.0 \mathrm{~cm}}$ and $\mathrm{BFI}_{2.5 \mathrm{~cm}}$, respectively. As a final quality check, the DCS dataset was discarded if $\mathrm{BFI}_{1.0 \mathrm{~cm}}>$ $\mathrm{BFI}_{2.5 \mathrm{~cm}}$. Here, we assume that $\mathrm{BFI}_{1 \mathrm{~cm}}$ primarily samples scalp and skull, whereas $\mathrm{BFI}_{2.5 \mathrm{~cm}}$ samples scalp, skull, and brain. Given that scalp and skull blood flow are known to be $\sim 1 / 6$ to $1 / 3$ lower than cerebral blood flow, ${ }^{42}$ it is certainly possible that $\mathrm{BFI}_{2.5 \mathrm{~cm}}$ may be lower than $\mathrm{BFI}_{1.0 \mathrm{~cm}}$ but still contain significant information about cerebral perfusion. However, we chose this rigorous cutoff to exclude datasets in which $\mathrm{BFI}_{2.5 \mathrm{~cm}}$ was obviously contaminated by the effects of extracerebral layers.

\subsection{Transcranial Doppler Ultrasound}

TCD examinations were performed by an experienced neurosonographer in a quiet atmosphere and without pharmacological sedation. A 2-MHz pulsed-wave Doppler probe (Sonara TD12019M, Carefusion, San Diego, California) was used to explore the major intracranial arteries through the temporal bone-window. The time averaged mean of the maximum velocity over an integral number of cardiac cycles was recorded bilaterally in the middle cerebral artery (MCA), anterior cerebral artery (ACA), and posterior cerebral artery. ${ }^{21,43}$

\subsection{Statistical Analysis}

Descriptive statistics were calculated for all variables of interest and included counts and percents or medians (interquartile range), as appropriate. Differences in demographic data were compared between SCD and control cohorts using Wilcoxon rank-sum tests (for continuous variables) or the Chi-square tests (for categorical variables). Paired Wilcoxon signed-rank tests were used to determine whether the DCS-measured BFI in sickle cell subjects differed from the age-/sex-matched healthy controls. Complete blood count parameters including hemoglobin, hematocrit, white blood cell, red blood cell, and reticulocyte counts, and TCD-measured mean blood flow velocities were associated with DCS-measured BFI within the SCD group using Spearman's correlation coefficient $\left(R_{s}\right)$. All statistical analyses were performed using the $R$ statistical packages (version 3.4.1, $\mathrm{R}$ Foundation), and statistical significance was assessed at the 0.05 level unless otherwise noted.

\section{Results}

Twenty-two children with SCD and 14 healthy controls were enrolled. One SCD subject withdrew before the start of DCS 
monitoring, and one SCD subject withdrew before the study started. Of the remaining 34 subjects, DCS data were excluded in eight SCD and two controls for low detected light intensity and one SCD for motion artifact. Further, one SCD subject and corresponding age-/sex-matched control were discarded because $\mathrm{BFI}_{1.0 \mathrm{~cm}}>\mathrm{BFI}_{2.5 \mathrm{~cm}}$ in the control. Thus, the final cohort consisted of 11 SCD patients and 11 age- and sexmatched healthy controls.

Patient characteristics of the final cohort are in Table 1. Each group consisted of four males and seven females who ranged in age from 5 to 8 years with a median age of 7 years. No significant differences in weight, height, BMI, or head circumference parameters were observed between groups. Of the 11 SCD subjects, seven had clinically indicated TCD evaluation within the same day as the optical measurement. No SCD subject had a history of overt stroke, while one SCD subject had a history of silent infarct. Of note, anatomical MRI scans were available in $n=3$ SCD subjects (ranging in age from 5.8 to 6.9 years, 2/1 $\mathrm{M} / \mathrm{F}$ ) within $\sim 1$ month of optical assessment. The average depth to the brain in this small subset of patients was $0.88 \pm 0.07 \mathrm{~cm}$.

$\mathrm{BFI}_{2.5 \mathrm{~cm}}$ was significantly elevated in sickle cell subjects compared to healthy age- and sex-matched controls [median (IQR): $3.16(2.75$ to 3.99$) \times 10^{-8}$ versus $1.12(0.76$ to 1.45$) \times$ $10^{-8} \mathrm{~cm}^{2} / \mathrm{s}, p<0.001$, Fig. 2(a)]. This difference between SCD and controls was not seen in $\mathrm{BFI}_{1.0 \mathrm{~cm}}\left[0.51[0.30\right.$ to 1.34$] \times 10^{-8}$ versus 0.23 [0.15 to 0.53$] \times 10^{-8} \mathrm{~cm}^{2} / \mathrm{s}, p=0.12$, Fig. 2(b)]. Given that the $\mathrm{BFI}_{1.0 \mathrm{~cm}}$ primarily reflects extracerebral (i.e., skull and scalp) blood flow, whereas the $\mathrm{BFI}_{2.5 \mathrm{~cm}}$ reflects a weighted combination of cerebral and extracerebral blood flow, these results suggest that the elevations in $\mathrm{BFI}_{2.5} \mathrm{~cm}$ in SCD subjects are driven by elevations in blood flow in the brain.

Within the sickle cell group, $\mathrm{BFI}_{2.5} \mathrm{~cm}$ was inversely correlated with hemoglobin level $\left(R_{s}=-0.75, p=0.012\right.$, Fig. 3), hematocrit $\left(R_{s}=-0.75, p=0.012\right)$, and red blood cell count $\left(R_{s}=-0.78, p=0.007\right)$ while positively correlated with age $\left(R_{s}=0.75, p=0.012\right)$, white blood cell count $\left(R_{s}=0.78\right.$, $p=0.007)$, and steady-state reticulocyte count $\left(R_{s}=0.82\right.$, $p=0.004)$. In contrast, $\mathrm{BFI}_{1.0 \mathrm{~cm}}$ did not correlate with age or any of the CBC parameters.

$\mathrm{BFI}_{2.5} \mathrm{~cm}$ positively correlated with TCD-measured cerebral blood flow velocity in MCA, but this trend did not reach
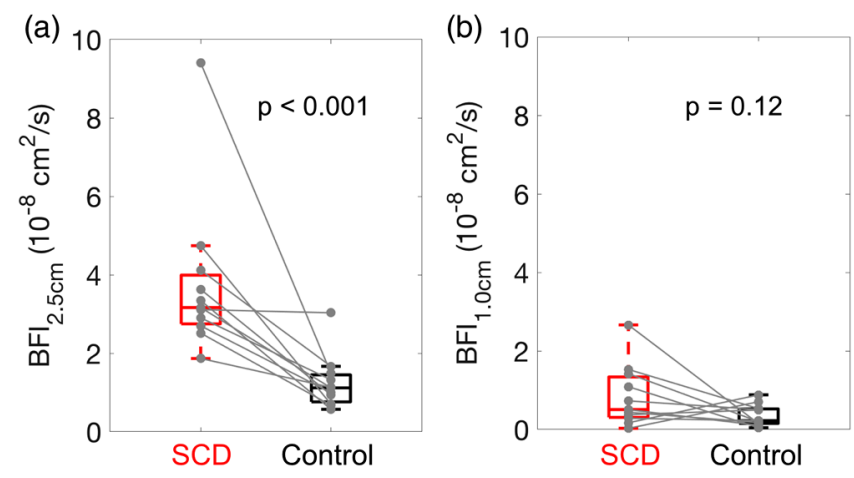

Fig. 2 Comparison of DCS measured BFI between age- and sexmatched sickle cell subjects (SCD, $n=11)$ and healthy controls $(n=11)$ at source-detection separation, (a) $r=2.5 \mathrm{~cm}\left(\mathrm{BFI}_{2.5 \mathrm{~cm}}\right)$ and (b) $1.0 \mathrm{~cm}\left(\mathrm{BFI}_{1.0} \mathrm{~cm}\right)$. For each boxplot, the central line denotes the median and the bottom and top edges of the box indicate the 25th and 75th percentiles, respectively. The whiskers extend to the most extreme data points not considered outliers. Individual connected dots represent each age- and sex-matched pair. statistical significance. $\left(R_{s}=0.68, p=0.11\right.$, Fig. 4) In contrast, $\mathrm{BFI}_{2.5 \mathrm{~cm}}$ was not significantly correlated with ACA ( $p=$ 0.96 , data not shown). Similar lack of correlation was observed between DCS-BFI ${ }_{1.0} \mathrm{~cm}$ and TCD measured blood flow velocities $\left(R_{s}=0.71\right.$ for MCA, $R_{s}=0.96$ for $\left.\mathrm{ACA}\right)$.

\section{Discussions}

Herein, we demonstrate the feasibility of DCS as a low-cost and noninvasive means to evaluate microvascular perfusion abnormalities in children with SCD. To our knowledge, this report is the first to apply DCS to this patient population. We found that the resting-state $\mathrm{BFI}_{2.5 \mathrm{~cm}}$ is significantly higher in sickle cell patients than age-/sex-matched healthy controls (Fig. 2). Further, within the sickle cell group, we found that $\mathrm{BFI}_{2.5} \mathrm{~cm}$ was inversely correlated with arterial hemoglobin level and hematocrit (Fig. 3). These observations are consistent with

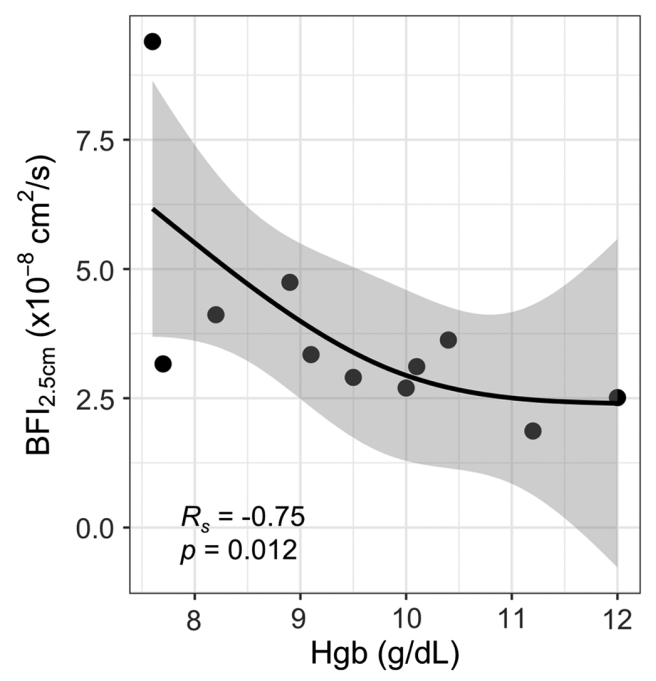

Fig. 3 DCS-measured BFI at $r=2.5 \mathrm{~cm}\left(\mathrm{BFI}_{2.5 \mathrm{~cm}}, \mathrm{~cm}^{2} / \mathrm{s}\right)$ as a function of arterial hemoglobin concentration $(\mathrm{g} / \mathrm{dL}, n=11)$. The BFI is significantly correlated with $\mathrm{Hgb}\left(R_{s}=-0.75, p=0.012\right)$. The solid line represents the best spline fit to the data and the gray-shaded area denotes the $95 \%$ confidence interval for the spline fit.

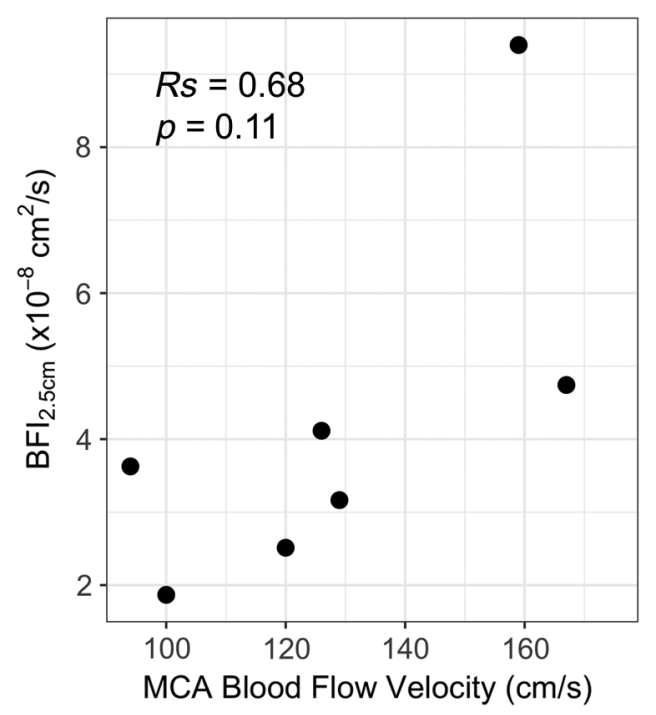

Fig. 4 Relationship between DCS-measured BFI at $r=2.5 \mathrm{~cm}$ and TCD-measured mean blood flow velocity in MCA $(\mathrm{cm} / \mathrm{s}),(n=7$, $\left.R_{s}=0.68, p=0.11\right)$. 
numerous other neuroimaging studies (MRI, PET, and Xenon inhalation) that suggest sickle patients experience persistent hyperemic vasodilation as a compensatory response to chronic anemia. ${ }^{14-16,27}$ Interestingly, we also observed significant correlations between $\mathrm{BFI}_{2.5 \mathrm{~cm}}$ and red blood cell, white blood cell, and steady-state reticulocyte counts (data not shown), although interpretation of these correlations should be met with caution given the high degree of association between these variables and hemoglobin concentration. In future studies with increased sample size, we will use multivariate regression or stratified models to explore the relative proportion of variance in $\mathrm{BFI}_{2.5 \mathrm{~cm}}$ that can be explained by each of these blood count measures.

Further, we observed a correlation between $\mathrm{BFI}_{2.5 \mathrm{~cm}}$ and TCD-measured blood flow velocity in middle cerebral arteries $\left(R_{s}=0.68\right.$, Fig. 4), suggesting that macro- and microvascular coupling likely remains intact for this cohort. We note that we were not adequately powered to demonstrate statistical significance given our small sample size $(n=7)$. This result contradicts prior studies using arterial spin-labeled (ASL) MRI that have reported a lack of correlation between tissue-level CBF and TCD-measured CBFV in SCD patients. ${ }^{13,29}$ This contradiction may be due to differences in age and disease severity between our relatively young and healthy cohort and those previously studied.

In total, our results suggest that DCS is qualitatively sensitive to the known increases in CBF associated with SCD and to the relationship of $\mathrm{CBF}$ to anemia and other associated hematological factors. In fact, DCS appears to quantitatively overestimate these blood flow abnormalities. While studies with MRI and PET have demonstrated $\sim 1.5 \times$ increases in CBF in SCD versus controls, ${ }^{14-16,27}$ we observed a markedly more pronounced three-fold difference in $\mathrm{BFI}_{2.5 \mathrm{~cm}}$ between sickle and control groups. Further, within the sickle cell group, the magnitude of increases in $\mathrm{CBF}$ with decreases in hemoglobin that we observed with DCS is much greater than expected. ${ }^{14-16,27}$ This added sensitivity may be advantageous in the application of DCS as a screening tool. In addition to enhanced sensitivity, DCS offers numerous other advantages over traditional neuroimaging modalities that measure tissue-level CBF. The manufacturing cost of a DCS system ( $\$ 40 \mathrm{k}$ for the system herein) is orders of magnitude lower than modalities such as MRI or PET. Further, DCS does not involve exposure to external contrast agent or ionizing radiation, and it is well tolerated in children of all ages without the need for sedation. Although DCS is limited in depth sensitivity to cortical tissue ${ }^{40}$ and in spatial sensitivity to frontal cortex (due to low SNR in the presence of hair), the cerebral effects of SCD appear to be global, affecting cortical as well as deep brain perfusion. ${ }^{12,14,16,27}$ Thus, DCS may provide a low cost, noninvasive tool to assess perfusion abnormalities that complements transcranial Doppler measures of macrovascular perfusion.

The apparent sensitivity of DCS-measured resting-state $\mathrm{BFI}_{2.5 \mathrm{~cm}}$ to cortical cerebral blood flow in this cohort is somewhat surprising. In general, the effects of extracerebral layers in DCS are thought to significantly confound cerebral influences on $\mathrm{BFI}_{2.5 \mathrm{~cm}}$ when using the semi-infinite solution to the correlation diffusion equation such that, to date, correlations between $\mathrm{BFI}_{2.5 \mathrm{~cm}}$ and CBF measured by other "gold standard" modalities have not been observed in pediatric cohorts $>1$ year. The sensitivity to brain of $\mathrm{BFI}_{2.5 \mathrm{~cm}}$ in these young patients is likely due to the relatively small average depth to the brain compared to our source detector separation $(0.9$ compared to $2.5 \mathrm{~cm})$.
However, sickle cell patients also exhibit reduced hematocrit and increased blood vessel diameter due to vasodilation, which should act to blunt expected differences in $\mathrm{BFI}_{2.5} \mathrm{~cm}$ seen in SCD compared to controls. ${ }^{38}$ Thus, a priori we would expect that, at best, $\mathrm{BFI}_{2.5 \mathrm{~cm}}$ would be elevated in sickle subjects compared to controls, but the degree of this elevation should be significantly lower than the $\sim 1.5 \times$ increase in CBF previously reported by both MRI and PET. ${ }^{14-16,27}$ On the contrary, as discussed above, DCS appears to overestimate $\mathrm{BFI}_{2.5} \mathrm{~cm}$ in SCD patients. The origin of the exaggerated $\mathrm{BFI}_{2.5 \mathrm{~cm}}$ differences seen in sickle cell patients is unclear; perhaps these differences arise from changes in the proportionality between $\mathrm{BFI}_{2.5 \mathrm{~cm}}$ and absolute $\mathrm{CBF}$ caused by pathophysiological changes in the cortex associated with SCD. ${ }^{38}$ Benchtop experiments in tissue-simulating phantoms and validation studies against ASL-MRI are ongoing to better understand these effects.

Finally, we note several limitations of our study. First, DCS data were discarded from a substantial fraction of subjects (10/ $32,31.2 \%$ ) due to poor SNR. However, we believe this low SNR was primarily caused by poor light transmission in our detector fiber bundle. After recruiting the first 22 subjects for this study and observing poor SNR in $10 / 22$, we remade our detector fiber, and we redesigned the sensor to protect the fibers from scratches and debris by coupling the fibers to right angle prisms (while maintaining source-detector geometry). Once we implemented this change, DCS data quality dramatically improved, with 10/10 subjects after redesign passing our quality criteria. Second, FDNIRS measurements of tissue optical properties $\left(\mu_{\mathrm{s}}^{\prime}\right.$ and $\left.\mu_{\mathrm{a}}\right)$ were not available for seven SCD patients and four controls. For these patients in which FDNIRS data were not available, group averages were used. Based on the observed variation of $\mu_{\mathrm{s}}^{\prime}$ across subjects, assumption of the group average $\mu_{\mathrm{s}}^{\prime}$ could induce errors ranging from $-30 \%$ to $55 \%$ in our estimation of BFI. However, given the $\sim 3 \times$ increase in $\mathrm{BFI}_{2.5} \mathrm{~cm}$ seen in SCD patients compared to controls, these errors should not be appreciable enough to qualitatively alter the main findings of this work. Nevertheless, future studies would benefit from simultaneous FDNIRS assessment of $\mu_{\mathrm{s}}^{\prime}$ and $\mu_{\mathrm{a}}$ and DCS in all subjects.

\section{Conclusion}

In summary, we noninvasively assessed resting-state cerebral blood flow in children with SCD using DCS for the first time. We found that the DCS-measured BFI at $2.5 \mathrm{~cm}$ is sensitive to increases in CBF associated with SCD. Further, we observed a correlation between DCS and TCD that suggests macro- and microvascular blood flow coupling remains intact in this cohort. These results suggest that DCS may provide a low-cost, noninvasive monitor of tissue-level cerebral blood flow in pediatric sickle cell patients, paving the way for future studies that explore the potential of DCS to detect perfusion abnormalities associated with stroke risk and/or cerebral vasculopathy.

\section{Appendix 1. FDNIRS Measurements}

To improve estimation of the DCS-measured BFI, it is ideal to measure the reduced scattering and absorption properties of the tissue ( $\mu_{\mathrm{s}}^{\prime}$ and $\mu_{\mathrm{a}}$, respectively). ${ }^{44}$ Thus, in a subset of patients, we used a customized commercial FDNIRS system (Imagent, ISS Inc., Illinois) to estimate $\mu_{\mathrm{s}}^{\prime}$ and $\mu_{\mathrm{a}}$. This system employs eight near-infrared laser diode sources $(690,730,750,775$, $785,800,825$, and $830 \mathrm{~nm})$ modulated at $110 \mathrm{MHz}$ and four photomultiplier tube detectors with gain modulation of 
$110 \mathrm{MHz}+5 \mathrm{kHz}$ to achieve heterodyne detection at $5 \mathrm{kHz}$. The patient interface consisted of a custom-designed threedimensional-printed FDNIRS sensor with one source optode and four detector optodes spaced 1.5, 2, 2.5, and $3 \mathrm{~cm}$ from the source in a black rigid holder. All NIRS source and detector optodes consisted of custom $2.5 \mathrm{~mm}$ fiber bundles of $50-\mu \mathrm{m}$ multimode fibers (FTTIIG23767, Fiberoptics Technology).

For FDNIRS data acquisition, the optical sensor was manually held over the right and left forehead sequentially. AC amplitude and phase data were acquired for $3 \mathrm{~s}$ at $20 \mathrm{~Hz}$. The sensor was repositioned three times over each hemisphere to reduce the influence of local inhomogeneities under the optical sensor.

For FDNIRS data analysis, we used the semi-infinite solution to the photon diffusion equation to relate the measured $\mathrm{AC}$ amplitude attenuation and phase shift as a function of source-detector separation to the wavelength-dependent $\mu_{\mathrm{s}}^{\prime}$ and $\mu_{\mathrm{a}}{ }^{45}$ For each measurement repetition, data were processed in an automated fashion using predefined, objective quality criteria as outlined in Ref. 46. First, raw data were discarded if the phase standard deviation exceeded two deg or if the AC amplitude ratio of standard deviation to mean was $>0.05$. Next, for each wavelength, the slopes of $\ln \left(A C \times r^{2}\right)$ and phase as functions of source detector separation, $r$, were used to estimate $\mu_{\mathrm{s}}^{\prime}$ and $\mu_{\mathrm{a}}$. Data from a given wavelength were discarded if $R^{2}<0.97$ for either linear fit. The entire dataset was discarded if $<6$ wavelengths total passed this linear fit criteria. Because DCS data were obtained at $852 \mathrm{~nm}$, we next extrapolated $\mu_{\mathrm{s}}^{\prime}$ and $\mu_{\mathrm{a}}$ at $852 \mathrm{~nm}$. To extrapolate $\mu_{\mathrm{a}}(852 \mathrm{~nm})$, the measured $\mu_{\mathrm{a}}(\lambda)$ was fit to the hemoglobin spectra (assuming 75\% water concentration and known extinction coefficients of oxy- and deoxyhemoglobin). To extrapolate $\mu_{\mathrm{s}}^{\prime}(852 \mathrm{~nm})$, we fit a linear model to the measured $\mu_{\mathrm{s}}^{\prime}(\lambda)$ as a function of wavelength. Finally, data were discarded if $p>0.01$ for the fits of either the measured $\mu_{\mathrm{a}}$ versus the expected hemoglobin spectrum or of the measured $\mu_{\mathrm{s}}^{\prime}$ versus wavelength.

FDNIRS measurements were only performed in a subset of 14/20 SCD and 9/14 controls. Data from 5/14 SCD subjects did not pass our predefined quality control criteria, resulting in nine SCD and nine controls used for FD-NIRS data analysis. We note that only 4/9 SCD and 8/9 controls with usable FDNIRS data also had usable DCS data. The estimated median [IQR] of $\mu_{\mathrm{s}}^{\prime}$ and $\mu_{\mathrm{a}}$ at $852 \mathrm{~nm}$ were $7.1\left[6.5\right.$ to $\left.8.5 \mathrm{~cm}^{-1}\right]$ and $0.13 \mathrm{~cm}^{-1}$ (a)

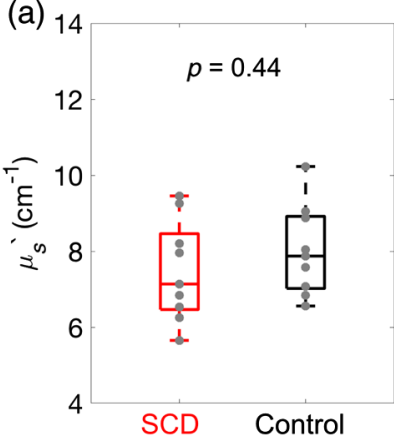

(b)

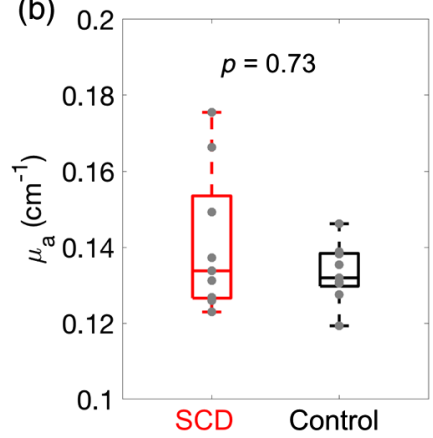

Fig. 5 Comparison of FDNIRS measured (a) reduced scattering coefficients, $\mu_{\mathrm{s}}^{\prime}$ and (b) absorption coefficients, $\mu_{\mathrm{a}}$ at $852 \mathrm{~nm}$ between sickle cell subjects (SCD, $n=9)$ and healthy controls $(n=9)$. For each boxplot, the central line denotes the median and the bottom and top edges of the box indicate the 25th and 75th percentiles, respectively. The whiskers extend to the most extreme data points not considered outliers. Individual dots represent each subject.
[0.13 to $0.15 \mathrm{~cm}^{-1}$ ] for SCD, and 7.9 [7.0 to $8.9 \mathrm{~cm}^{-1}$ ] and 0.13 [0.13 to $0.14 \mathrm{~cm}^{-1}$ ] for healthy controls. Neither $\mu_{\mathrm{s}}^{\prime}$ nor $\mu_{\mathrm{a}}$ were significantly different between two groups (Fig. 5). No differences were observed between right and left hemispheres. Further, within SCD cohort, no trends were observed between $\mu_{\mathrm{s}}^{\prime}$ and any of the CBC parameters. In contrast, $\mu_{\mathrm{a}}$ at $852 \mathrm{~nm}$ showed significant correlations with hemoglobin $\left(R_{s}=0.83, p=0.008\right)$ and hematocrit $\left(R_{s}=0.8, p=0.014\right)$, marginal correlation with red blood cell $\left(R_{s}=0.67, p=\right.$ 0.059 ), and no correlations with white blood cell or reticulocyte counts.

\section{Disclosures}

No conflicts of interest, financial or otherwise, are declared by the authors.

\section{Acknowledgments}

We thank Amanda Watts, Morgan Barnett, and Gabrielle Dean for their clinical support. We also thank Dr. R. Clark Brown for clinical insights and Zhou Fang for technical support on sensor construction. This project was supported by the National Institutes of Health R21-HL138062 (EMB), Children's Healthcare of Atlanta Junior Faculty Focused Award (EMB), and the American Heart Association Postdoctoral Fellowship 19POST34380337 (SYL).

\section{References}

1. F. B. Piel, M. H. Steinberg, and D. C. Rees, "Sickle cell disease," N. Engl. J. Med. 376(16), 1561-1573 (2017).

2. D. C. Rees, T. N. Williams, and M. T. Gladwin, "Sickle-cell disease," Lancet 376(9757), 2018-2031 (2010).

3. M. R. DeBaun et al., "Silent cerebral infarcts: a review on a prevalent and progressive cause of neurologic injury in sickle cell anemia," Blood 119(20), 4587-4596 (2012).

4. F. Bernaudin et al., "Impact of early transcranial Doppler screening and intensive therapy on cerebral vasculopathy outcome in a newborn sickle cell anemia cohort," Blood 117(4), 1130-1140; quiz 1436 (2011).

5. F. Bernaudin et al., "Chronic and acute anemia and extracranial internal carotid stenosis are risk factors for silent cerebral infarcts in sickle cell anemia," Blood 125(10), 1653-1661 (2015).

6. C. J. Earley et al., "Stroke in children and sickle-cell disease: BaltimoreWashington Cooperative Young Stroke Study," Neurology 51(1), 169-176 (1998).

7. C. T. Hijmans et al., "Neurocognitive deficits in children with sickle cell disease: a comprehensive profile," Pediatr. Blood Cancer 56(5), 783-788 (2011)

8. J. Schatz et al., "Poor school and cognitive functioning with silent cerebral infarcts and sickle cell disease," Neurology 56(8), 1109-1111 (2001)

9. W. Wang et al., "Neuropsychologic performance in school-aged children with sickle cell disease: a report from the cooperative study of sickle cell disease," J. Pediatr. 139(3), 391-397 (2001).

10. A. M. Behpour et al., "Cerebral blood flow abnormalities in children with sickle cell disease: a systematic review," Pediatr. Neurol. 48(3), 188-199 (2013).

11. K. C. Wood, L. L. Hsu, and M. T. Gladwin, "Sickle cell disease vasculopathy: a state of nitric oxide resistance," Free Radical Biol. Med. 44(8), 1506-1528 (2008)

12. S. Gevers et al., "Arterial spin labeling measurement of cerebral perfusion in children with sickle cell disease," J. Magn. Reson. Imaging 35(4), 779-787 (2012).

13. J. J. Strouse et al., "Inverse correlation between cerebral blood flow measured by continuous arterial spin-labeling (CASL) MRI and neurocognitive function in children with sickle cell anemia (SCA)," Blood 108(1), 379-381 (2006)

14. I. Prohovnik et al., "Cerebral hyperemia, stroke, and transfusion in sickle cell disease," Neurology 39(3), 344 (1989). 
15. A. M. Bush et al., "Determinants of resting cerebral blood flow in sickle cell disease," Am. J. Hematol. 91(9), 912-917 (2016).

16. M. E. Fields et al., "Regional oxygen extraction predicts border zone vulnerability to stroke in sickle cell disease," Neurology 90(13), E1134 (2018).

17. Y. S. Kim et al., "Dynamic cerebral autoregulation in homozygous Sickle cell disease," Stroke 40(3), 808-814 (2009).

18. E. Nur et al., "Cerebrovascular reserve capacity is impaired in patients with sickle cell disease," Blood 114(16), 3473-3478 (2009).

19. I. Prohovnik et al., "Hemodynamic etiology of elevated flow velocity and Stroke in sickle-cell disease," J. Cereb. Blood Flow Metab. 29(4), 803-810 (2009).

20. M. L. Hulbert and A. L. Ford, "Understanding sickle cell brain drain," Blood 124(6), 830-831 (2014).

21. R. Adams et al., "The use of transcranial ultrasonography to predict stroke in sickle cell disease," N. Engl. J. Med. 326(9), 605-610 (1992)

22. R. J. Adams et al., "Prevention of a first stroke by transfusions in children with sickle cell anemia and abnormal results on transcranial Doppler ultrasonography," N. Engl. J. Med. 339(1), 5-11 (1998).

23. L. B. Goldstein et al., "Primary prevention of ischemic stroke: a statement for healthcare professionals from the Stroke Council of the American Heart Association," Stroke 32(1), 280-299 (2001).

24. R. J. Adams et al., "Stroke and conversion to high risk in children screened with transcranial Doppler ultrasound during the STOP study," Blood 103(10), 3689-3694 (2004).

25. R. J. Adams, "Lessons from the stroke prevention trial in sickle cell anemia (STOP) study," J. Child Neurol. 15(5), 344-349 (2000).

26. R. J. Adams, "Big strokes in small persons," Arch. Neurol. 64(11), 1567-1574 (2007).

27. S. Herold et al., "Measurement of regional cerebral blood flow, blood volume and oxygen metabolism in patients with sickle cell disease using positron emission tomography," Stroke 17(4), 692-698 (1986).

28. X. W. van den Tweel et al., "Cerebral blood flow measurement in children with sickle cell disease using continuous arterial spin labeling at 3.0-Tesla MRI," Stroke 40(3), 795-800 (2009).

29. P. L. Croal et al., "Assessment of cerebral blood flow with magnetic resonance imaging in children with sickle cell disease: a quantitative comparison with transcranial Doppler ultrasonography," Brain Behav. 7(11), e00811 (2017).

30. F. J. Kirkham et al., "Perfusion magnetic resonance abnormalities in patients with sickle cell disease," Ann. Neurol. 49(4), 477-485 (2001).

31. K. P. Guilliams et al., "Red cell exchange transfusions lower cerebral blood flow and oxygen extraction fraction in pediatric sickle cell anemia," Blood 131(9), 1012-1021 (2018).

32. W. C. Wang et al., "Multicenter comparison of magnetic resonance imaging and transcranial Doppler ultrasonography in the evaluation of the central nervous system in children with sickle cell disease," J. Pediatr. Hematol. Oncol. 22(4), 335-339 (2000).

33. D. A. Boas, L. E. Campbell, and A. G. Yodh, "Scattering and imaging with diffusing temporal field correlations," Phys. Rev. Lett. 75(9), 1855-1858 (1995).

34. D. A. Boas and A. G. Yodh, "Spatially varying dynamical properties of turbid media probed with diffusing temporal light correlation," J. Opt. Soc. Am. A 14(1), 192-215 (1997).

35. E. M. Buckley et al., "Diffuse correlation spectroscopy for measurement of cerebral blood flow: future prospects," Neurophotonics 1(1), 011009 (2014).

36. T. Durduran and A. G. Yodh, "Diffuse correlation spectroscopy for non-invasive, micro-vascular cerebral blood flow measurement," Neuroimage 85(Part 1), 51-63 (2014).

37. T. Durduran et al., "Diffuse optics for tissue monitoring and tomography," Rep. Prog. Phys. 73(7), 076701 (2010).

38. D. A. Boas et al., "Establishing the diffuse correlation spectroscopy signal relationship with blood flow," Neurophotonics 3(3), 031412 (2016).

39. www.correlator.com

40. J. Selb et al., "Sensitivity of near-infrared spectroscopy and diffuse correlation spectroscopy to brain hemodynamics: simulations and experimental findings during hypercapnia," Neurophotonics 1(1), 015005 (2014).

41. E. Sathialingam et al., "Small separation diffuse correlation spectroscopy for measurement of cerebral blood flow in rodents," Biomed. Opt. Express 9(11), 5719-5734 (2018).
42. E. Ohmae et al., "Cerebral hemodynamics evaluation by near-infrared time-resolved spectroscopy: correlation with simultaneous positron emission tomography measurements," Neuroimage 29(3), 697-705 (2006)

43. R. Aaslid, "Transcranial Doppler examination techniques," in Transcranial Doppler Sonography, R. Aaslid, Ed., pp. 39-59, Springer, Vienna (1986).

44. D. Irwin et al., "Influences of tissue absorption and scattering on diffuse correlation spectroscopy blood flow measurements," Biomed. Opt. Express 2(7), 1969-1985 (2011).

45. S. Fantini et al., "Frequency-domain multichannel optical-detector for noninvasive tissue spectroscopy and oximetry," Opt. Eng. 34(1), 32-42 (1995).

46. N. Roche-Labarbe et al., "Noninvasive optical measures of CBV, $\mathrm{StO}_{2}, \mathrm{CBF}$ index, and $\mathrm{rCMRO}_{2}$ in human premature neonates' brains in the first six weeks of life," Hum. Brain Mapp. 31(3), 341-352 (2010)

Seung Yup Lee is an American Heart Association postdoctoral fellow in biomedical engineering at Georgia Tech and Emory University. $\mathrm{He}$ received his $\mathrm{PhD}$ in biomedical engineering at the University of Michigan, Ann Arbor, USA. His research focuses on quantitative tissue optical imaging and sensing for clinical applications.

Kyle R. Cowdrick is a doctoral candidate in biomedical engineering at Georgia Tech and Emory University. He received his master's degree in bioengineering innovation and design from the Johns Hopkins University in May 2017 and is a founding member of SpineAlign Surgical LLC. He is active in Georgia Tech's BME Capstone program. His current research focuses on early-stage clinical feasibility studies to assess the translational potential of diffuse optics in pediatric cardiac arrest and traumatic brain injury.

Bharat Sanders is an MD candidate at the Medical College of Georgia. He graduated from the Georgia Institute of Technology with a BS degree in biomedical engineering in May 2016. His research interests include biomedical devices, emergency medicine, and pediatrics.

Eashani Sathialingam is a doctoral student in biomedical engineering at Georgia Institute of Technology and Emory University. She received her bachelor's degree in biomedical engineering at the University of California, Irvine. Her research is focused on developing algorithms and applying diffuse optical technologies for in vitro and in vivo studies.

Courtney E. McCracken is the director of the Pediatric Biostatistics Core in the Department of Pediatrics at Emory University. The Pediatric Biostatistics Core supports clinical, translational, and basic science across both Emory University and Children's Healthcare of Atlanta. Her research interests include clinical trials, survival analysis, and mixed models.

Wilbur A. Lam, MD, PhD has a unique background as a physicianscientist-engineer trained in clinical pediatric hematology/oncology and bioengineering. His overall research interests involve developing and applying micro/nanosystems to advance biomedical research with a focus on the cellular mechanics of hematologic processes. In particular, his laboratory has a successful track record in developing microfluidic technologies as research-enabling platforms to study the biophysics of cell-cell interactions in blood disorders such as sickle cell disease.

Clinton H. Joiner: Biography is not available.

Erin M. Buckley is an assistant professor in the Wallace H. Coulter Department of Biomedical Engineering at Georgia Institute of Technology/Emory University and the Department of Pediatrics at Emory University. She received her PhD from the Department of Physics and Astronomy from the University of Pennsylvania in 2011 and completed postdoctoral training at the Children's Hospital of Philadelphia and at Massachusetts General Hospital. Her research focuses on the development diffuse optical spectroscopies for brain monitoring. 